\title{
Green Synthesis and Characterization of Ag@Au Core-shell Bimetallic Nanoparticles using the Extract of Hamelia patens Plant
}

\author{
K. Chavez ${ }^{1 *}$ and G. Rosas ${ }^{1}$. \\ ${ }^{1 .}$ Instituto de investigación en Metalurgia y Materiales, UMSNH, Morelia, México. \\ * Corresponding author: kchavez@umich.mx
}

The noble metals nanoparticles (Nps) have a great interest due to their excellent properties and as a consequence their wide variety of applications in which they are actively used [1,2]. The bimetallic nanoparticles are of great interest because the synergic properties that improve their applications [3, 4]. In this work, we present the synthesis of bimetallic Ag-Au nanoparticles, using the Hamelia Patens plant extract as a reducing and stabilizing agent.

Ag seeds were used for the synthesis of the bimetallic Nps through heterogeneous nucleation. During the experimental procedure, the extract concentration of $2 \% \mathrm{w} / \mathrm{v}$ (weight/volume) in $100 \mathrm{ml}$ of distilled water was used. The solution to prepare the infusion was prepared with magnetic stirring and a temperature of $50^{\circ} \mathrm{C}$. Firstly; it was synthesized silver seeds using $2 \% \mathrm{w} / \mathrm{v}$ plant and $12 \mathrm{mM} \mathrm{AgNO}_{3}$. Then the gold salt $\left(5 \mathrm{mM} \mathrm{HAuCl}_{4}\right)$ is added with volumetric ratio 1: 1: 0.5 , extract, silver salt and gold salt respectively.

Figure 1a shows the results analyzed by the Uv-vis technique. It is appreciated a plasmon peak located at around $550 \mathrm{~nm}$, also showing a relatively good particle size distribution. To evaluate this result as regards the possibility of obtaining core-shell Nps. It must be remembered that the peak of the plasmon corresponding to $\mathrm{Ag} \mathrm{Nps}$ is characteristic at $450 \mathrm{~nm}$, so that, if it is located in that position it would be counting on independent Ag and Au Nanoparticles. Figure 1b displays the IR spectra of the synthesized systems, starting with the spectrum of the extract (Fig. 1b black line), the silver Nps (Fig. 1b red line), and finally the synthesis of bimetallic Nps Ag-Au (Fig. 1b blue line). The band of the $-\mathrm{OH}$ in $3420 \mathrm{~cm}^{-1}$ decreases as the reaction is carried out. It is observed that the carbonyls bands located in 2926 and 1622 $\mathrm{cm}^{-1}$ reduction in intensity for the bimetallic Nps. The phenols band situated at 1257 and $779 \mathrm{~cm}^{-1}$ tend to disappear in the case of mono and bimetallic Nps. The peak that corresponds to the $-\mathrm{CH}$ located at $1432 \mathrm{~cm}^{-1}$ only decreases at the intensity. The alcohols band at $1062 \mathrm{~cm}^{-1}$ is decreasing with the nanoparticles synthesis; also, the amine peak at $660 \mathrm{~cm}^{-1}$ decreases intensity after biosynthesis. Thus, the functional groups responsible for the bioreduction were phenols and carbonyls. In the same way, the groups $-\mathrm{OH}$ and $-\mathrm{CH}$ were attributed to the nanoparticle stabilization.

To continue the characterization of the nanoparticles, micrographs in figures 2a-b) show that the metallic particles are less than $100 \mathrm{~nm}$, although it is difficult to appreciate by this technique if the Nps correspond to a core-shell configuration, it is also observed a homogeneous distribution and spherical Nanoparticles. The chemical analysis by EDS was carried out (Fig. 2c), showing the elements silver, gold and chlorine.

Figure 3a shows a bright-field image illustrating the particle size distribution ranging from 10 to $50 \mathrm{~nm}$ being the average particle size is $32 \mathrm{~nm}$. Figure $3 \mathrm{~b}$ displays a high-resolution image (HRTEM) of a bimetallic nanoparticle. Several small crystalline regions randomly disoriented are observed surround the Nps, which indicates its polycrystallinity. These results are related with relatively fast aggregation rate of the atoms on the Nps. The structure of the nanoparticles can only be explained from the 
polynuclear growth model on the surface. Figure 3c displays the XRD pattern indexed with the silver and gold elements according to the PDF database (JCPDS \# 87-0719 and JCPDS \# 65-2870). It is important to point out that both elements have approximately the same lattice parameters due to their similar atomic radius and crystalline lattice (fcc). However, it can be observed an apparent broadening of the diffraction peak from which the presence of crystals of nanometric order can be derived. Besides, silver chloride (JCPDS \# 85-1355) is found as an unwanted byproduct. The studies showed that the Hamelia Patens plant presents the appropriate organic substances to carry out the reduction at a reasonably fast rate [5].

References:

[1] A Ongartkit, S Ananta and L Srisombat, Chemical Physics Letters 605-606 (2014), p. 85.

[2] F Hubenthal, Comprehensive Nanoscience and Technology, p. 375. http://dx.doi.org/10.1016/B978-0-12-

374396-1.00034-9(2011).

[3] K Gajanan and SN Tijare, Materials Today: Proceedings 5 (2018), p. 1093.

[4] CNR Rao, A Müller and AK Cheetham in "The Chemistry of Nanomaterials: Synthesis, Properties and Applications", (Wiley).

[5] The author K. Chavez gratefully acknowledges financial support to CONACYT and the UJAT. To the UMNSNH for the use of the facilities.

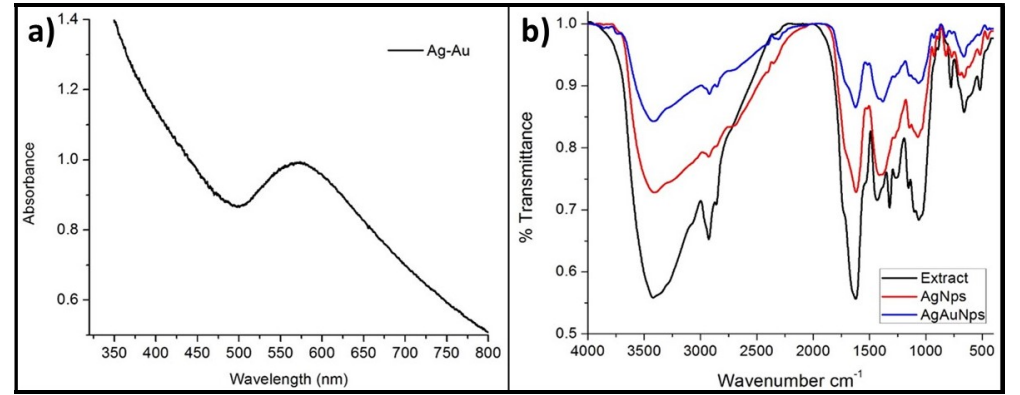

Figure 1. (a) Uv-vis spectra of Ag-Au bimetallic nanoparticles, (b) FTIR spectra of Hamelia Patens extract, Ag Nps and Ag-Au nanoparticles.

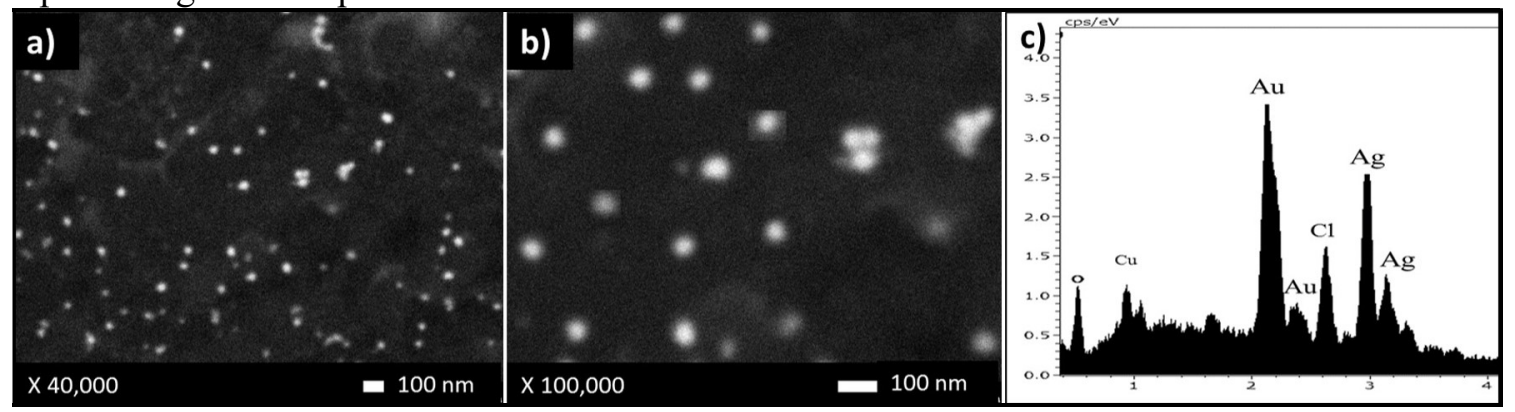

Figure 2. SEM images (a) 40,000 X, (b) $100,000 \mathrm{X}$ and (c) EDS.

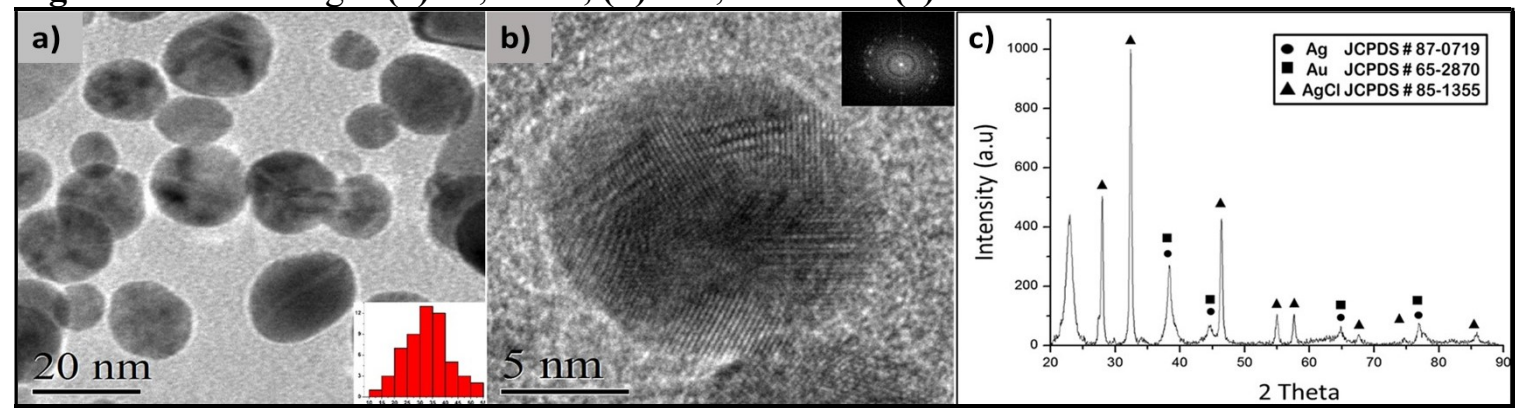

Figure 3. TEM images and size distribution (a) bright field, (b) HRTEM of core Ag and Au shell Nps and, (c) XRD pattern of Ag-Au bimetallic nanoparticles. 